INPLASY

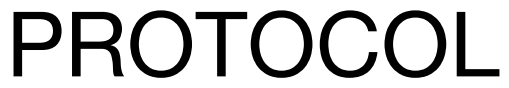

To cite: Gao et al. Acupuncture combined with SSRIs in the treatment of perimenopausal depressive disorder: $\mathrm{A}$ protocol for systematic review and meta-analysis. Inplasy protocol 2021120080. doi: 10.37766/inplasy2021.12.0080

Received: 17 December 2021

Published: 17 December 2021

Corresponding author: Zhan Gao

1151712376@qq.com

Author Affiliation:

Shandong University of

Traditional Chinese Medicine.

Support: NCFC.

Review Stage at time of this submission: The review has not yet started.

Conflicts of interest: None declared.

\section{Acupuncture combined with SSRIs in the treatment of perimenopausal depressive disorder: A protocol for systematic review and meta-analysis}

Gao, Z1; Wang, JQ2; Gao, M³; Song, T4; Gao, X5; Gao, J6.

Review question / Objective: This study evaluated the effectiveness and safety of acupuncture combined with SSRIs in the treatment of Perimenopausal depressive disorder by Meta-analysis.

Condition being studied: Perimenopausal depressive disorder is an affective disorder involving endocrine, neurological, immune, which seriously endangers the physical and mental health of human beings.Serotonin Reuptake Inhibitor is the current first-line clinical treatment, has limited efficacy and serious side effects. Acupuncture combined with SSRIs therapy has been widely used clinically because it increases efficacy and reduces side effects. There is a lack of highquality evidence to assess its efficacy and safety.

INPLASY registration number: This protocol was registered with the International Platform of Registered Systematic Review and Meta-Analysis Protocols (INPLASY) on 17 December 2021 and was last updated on 17 December 2021 (registration number INPLASY2021120080).

\section{INTRODUCTION}

Review question / Objective: This study evaluated the effectiveness and safety of acupuncture combined with SSRIs in the treatment of Perimenopausal depressive disorder by Meta-analysis.
Condition being studied: Perimenopausal depressive disorder is an affective disorder involving endocrine, neurological, immune, which seriously endangers the physical and mental health of human beings.Serotonin Reuptake Inhibitor is the current first-line clinical treatment, has limited efficacy and serious side effects. Acupuncture 
combined with SSRIs therapy has been widely used clinically because it increases efficacy and reduces side effects. There is a lack of high-quality evidence to assess its efficacy and safety.

\section{METHODS}

Participant or population: Women were diagnosed with PDD. The diagnostic criteria for Depressive Disorder were in accordance with the Chinese Classification and Diagnostic Criteria of Mental Disorders, 3rd edition (CCMD-3), American Psychiatric Association DSM-5,American Psychiatric Association's Diagnostic and Statistical Manual of Mental Disorders; diagnostic criteria for perimenopause in accordance with the Diagnostic Criteria for Gynecological Diseases, the diagnostic criteria for TCM evidence should be in accordance with the "Internal Medicine of Chinese Medicine" or "Diagnostic and Efficacy Criteria for TCM Medical Evidence".

Intervention: All patients used received acupuncture combined with SSRIs in the experimental group. The SSRI drugs were same as the control group.

Comparator: Patients used were treated with SSRIs in the control group.

Study designs to be included: We will include clinical randomized controlled trials (RCTs), which will be categorized by language in Chinese and English. Any other types of studies, such as animal studies, case reports, case series and reviews, will be excluded.

Eligibility criteria: The PICOS (participant, intervention, comparison, and study design) principle has been applied in the study design.

Information sources: From the construction of the library to December 16, 2021, all randomized controlled trials articles about acupuncture combined with SSRIs treatment of PDD will be searched in databases, such as PubMed, Cochrane
Library, Web of sicence, China National Knowledge Infrastructure, Wan Fang, Wei Pub by advanced search. The key search terms used are (Perimenopause or Climacteric or Menopause) And (Depressive Disorder or Mood Disorders) And (Serotonin Uptake Inhibitors or Fluoxetine or Paroxetine or Sertraline or FIuvoxamine or Citalopram or Escitalopram) And (Acupuncture or manual acupuncture or electroacupuncture or scalp acupuncture or auricular acupuncture).

Main outcome(s): The primary outcome are the Hamilton depressive disorder scale (HAMD) and Clinical efficiency.

Additional outcome(s): The primary Secondary outcome are the Self-rating depressive disorder scale (SDS), quality of life scale (SF-36) and Estradiol levels.

Quality assessment / Risk of bias analysis: The Cochrane Collaboration tool was used to assess the risk of bias in randomised trials, including the generation of randomised sequences, allocation concealment, implementation of blinding (blinding of subjects and implementers and blinding of study outcomes), data integrity, selective publication and other biases, all of which could be classified as 'low risk', 'unclear' and 'high risk'. "unclear" and "high risk"19. After two authors have completed their assessments, the results are cross-checked if there is disagreement, a third author discusses arbitration.

Strategy of data synthesis: We will use Review Manager Software (RevMan) V. 5.4 (Copenhagen, Denmark) for data analysis and quantitative data synthesis. If no statistical heterogeneity is found, a fixedeffects model is used for data synthesis. If there is significant statistical heterogeneity, we will use a random effects model.

Subgroup analysis: When heterogeneity occurs in the analysis, different drugs in SSRIs can be analyzed in subgroups. 
Sensitivity analysis: Sensitivity analysis was performed to exclude tests with quality defects and to ensure the stability of analytical results based on sample size, study design, heterogeneity, methodological quality, and statistical models.

Country(ies) involved: China.

Keywords: acupuncture, selective serotonin reuptake inhibitors; permenopausal depressive disorder; perimenopausal period, depressive disorder; Meta-analysis; protocol; systematic review.

Contributions of each author:

Author 1 - Zhan Gao.

Author 2 - Jie Qiong Wang.

Author 3 - Mingzhou Gao.

Author 4 - Tingting Song.

Author 5 - Xiangju Gao.

Author 6 - Jie Gao. 